\title{
Automatic Generation Control Scheme In an Inter Connected Power System Using PSO Optimized Smes and Tcps
}

\author{
Geoffrey Eappen, AlokSahu \\ Research Scholar ME (DTI-Electrical)SGSITS Indore(M.P) \\ Research Scholar M. Tech. (Electronics)SGGSIE\&T Nanded (M.S.)
}

\begin{abstract}
In this paper an attempt has been made to control frequency deviations using Particle Swarm Optimization in a two area system. And PSO technique has been used to optimize the parameters of TCPS and SMES. Here TCPS is installed in series with Tieline in coordination with SMES. There is the comparative study of transient response and tie-line deviation of the system with and without optimized TCPS and SMES. The proposed frequency scheme is improved by using PSO optimized SMES and TCPS.

Keywords: Automatic Generation Control (AGC), Particle Swarm Optimization (PSO), Automatic Load frequency control (ALFC), Super conducting magnetic energy storage system (SMES), Thyristor controlled phase shifter(TCPS).
\end{abstract}

\section{INTRODUCTION}

The main objective of power system operation and control is to maintain continuous supply of power with an acceptable quality, to all the consumers in the system. The system will be in equilibrium, when there is a balance between the power demand and the power generated. There are two basic control mechanisms used to achieve reactive power balance (acceptable voltage profile) and real power balance (acceptable frequency values).The former is called the automatic voltage regulator (AVR) and the latter is called the automatic load frequency control (ALFC) or automatic generation control (AGC)[1].

The parallel operation of interconnected systems is the today's requirement with the increase of size of electric power system, controlling the frequency of interconnected power system has becoming the challenge for control engineer[3]. The deviation of the frequencies and tie-line power arise because of unpredictable load variations, which occur due to a mismatch between the generated and the demandedpower[1].The main objective of providing an Automatic Generation Control is to maintain system frequency at nominal value.

\section{A.Automatic Generation Control:Fundamentals}

The main objectives of AGC are to regulate the frequency (using both primary and supplementary controls). The ALFC is use to control the frequency deviation by maintaining the real power balance in the system. The ALFC loop shown in Fig, is called the primary ALFC loop. It achieves the primary goal of real power balance by adjusting the turbine output to match the change in load demand[3]. The restoration of the frequency to the nominal value requires an additional control loop called the supplementary loop.This objective is met by using integral controller which makes the frequency deviation zero[2].The ALFC with the supplementary loop is generally called the AGC.

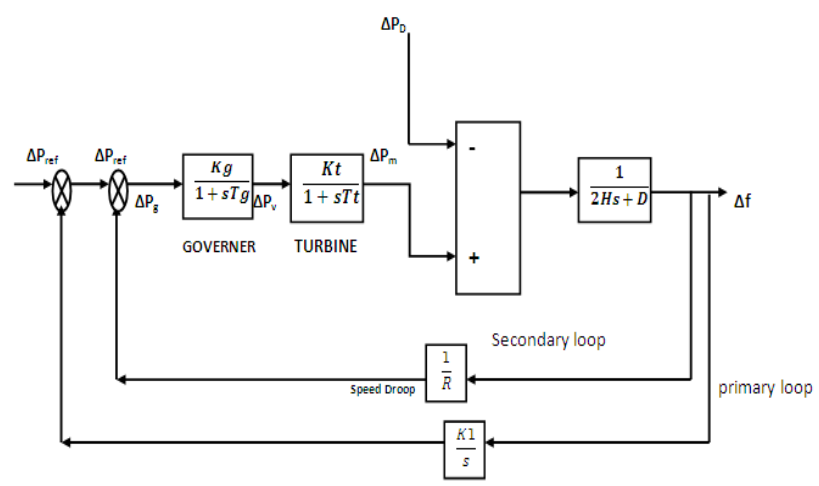

Fig1:Block diagram representation of AGC unit.

In a single area system, there is no tie-line schedule to be maintained.Only it has to deal with frequency. 


\section{B.Modeling of TCPS:}

The incremental Tie -Line power flow without TCPS is given as[5]:

$$
\Delta P_{\text {tie12 }}(s)=\frac{2 \pi T_{12}}{s}\left(\Delta F_{1}(s)-\Delta F_{2}(s)\right)
$$

Where $\mathrm{T}_{12}$ is the synchronizing constant without TCPS and $\Delta \mathrm{F}_{1}(\mathrm{~s}), \Delta \mathrm{F}_{2}(\mathrm{~s})$ are the frequency deviation in area1 \& area2 respectively.Current flowing from Area 1 to Area 2 is given by[5], when TCPS is placed in cascade form with tie-line

$i_{12}=\left\{\left|V_{1}\right| \angle\left(\delta_{1}+\phi\right)-\left|V_{2}\right| \angle\left(\delta_{2}\right)\right\} j X_{12}$

and

$$
P_{\text {tiel2 }}-j Q_{\text {tiel2 }}=\left|V_{1}\right| \angle-\left(\delta_{1}+\phi\right)\left\{\left|V_{1}\right| \angle\left(\delta_{1}+\phi\right)-\left|V_{2}\right| \angle\left(\delta_{2}\right)\right\} / j X_{12}
$$

The above expression can be written as

$$
\begin{aligned}
& P_{\text {tiel2 } 2}=\frac{\left|V_{1}\right|\left|V_{2}\right|}{X_{12}} \sin \left(\delta_{1}-\delta_{2}+\phi\right) \\
& \Delta P_{\text {tiel2 }}=\frac{\left|V_{1}\right|\left|V_{2}\right|}{X_{12}} \cos \left(\delta_{1}^{0}-\delta_{2}^{0}+\phi^{0}\right) \sin \left(\Delta \delta_{1}-\Delta \delta_{2}+\Delta \phi\right)
\end{aligned}
$$

In the above equation, $\Delta \delta_{1}-\Delta \delta_{2}+\Delta \phi$ can be neglected, therefore,

$$
\begin{aligned}
& \Delta P_{\text {tie12 }}=\frac{\left|V_{1}\right|\left|V_{2}\right|}{X_{12}} \cos \left(\delta_{1}^{0}-\delta_{2}^{0}+\phi^{0}\right)\left(\Delta \delta_{1}-\Delta \delta_{2}+\Delta \phi\right)(6) \\
& \Delta P_{\text {tie12 }}=\frac{\left|V_{1}\right|\left|V_{2}\right|}{X_{12}} \cos \left(\delta_{1}^{0}-\delta_{2}^{0}+\phi^{0}\right) \sin \left(\Delta \delta_{1}-\Delta \delta_{2}+\Delta \phi\right) \\
& \Delta P_{\text {tie12 }}=T_{12}\left(\Delta \delta_{1}-\Delta \delta_{2}+\Delta \phi\right)
\end{aligned}
$$

Where, $T_{12}=\frac{\left|V_{1}\right|\left|V_{2}\right|}{X_{12}} \cos \left(\delta_{1}^{o}-\delta_{2}^{o}+\phi^{o}\right)$

$$
\Delta P_{\text {tie12 }}=T_{12}\left(\Delta \delta_{1}-\Delta \delta_{2}\right)+T_{12} \Delta \phi
$$

But $\Delta \delta_{1}=2 \Pi \int \Delta f_{1} d t$ and $\Delta \delta_{2}=2 \Pi \int \Delta f_{2} d t$

Therefore,

$$
\Delta P_{\text {tiel2 }}=2 \Pi T_{12}\left(\int \Delta f_{1} d t-\int \Delta f_{2} d t\right)+T_{12} \Delta \phi
$$

Its Laplace transform given by

$$
\Delta P_{\text {tie12 }}=\frac{2 \Pi T_{12}}{s}\left(\Delta F_{1}(s) d t-\Delta F_{1}(s) d t\right)+T_{12} \Delta \phi(s)
$$

The tie line power flow can be controlled by the phase shifter angle $\Delta \phi$.

Assuming the control input signal to the TCPS controller is $\triangle E r r o r(s)$ and that the transfer function of the signaling conditioning circuit is $\mathrm{K}_{\phi}(\mathrm{s})$, where $\mathrm{K}_{\phi}(\mathrm{s})$ is the gain of the TCPS controller.

The phase shifter angle can be written as

$$
\Delta \phi(s)=\frac{K_{\phi}}{1+s T_{p s}} \Delta \operatorname{Error}(s)
$$

Where $\mathrm{K}_{\phi}$ and $\mathrm{T}_{\mathrm{ps}}$ are the gain and the time constants of the TCPS and $\triangle$ Error $_{l}(s)$ is the control signals which control phase angleof the phase shifter.

Thus Eqn.12 can be written as :

$\Delta P_{\text {tiel2 }}=\frac{2 \Pi T_{12}}{s}\left(\Delta F_{1}(s) d t-\Delta F_{1}(s) d t\right)+T_{12} \frac{K_{\phi}}{1+s T_{p s}} \Delta \operatorname{Error}(s)(14)$

$\Delta$ Error 1 can be any signal such as the areafrequency deviation $\Delta f_{1}$ or frequency deviation $\Delta f_{2}$ orACE of the other area to the TCPS unit tocontrol the TCPS phase shifter angle which in turncontrols the tie-line power flow. 
The above logic can be demonstrated as below:

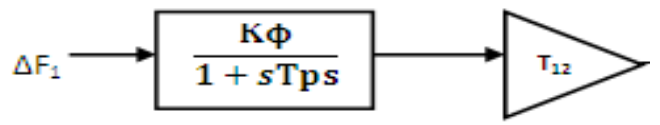

Fig1:Structure of TCPS as a frequency stabilizer

\section{C.Particle Swarm Optimization (PSO):}

Particle Swarm Optimization (PSO) is a technique used to explore the search space of a given problem to find the settings or parameters required to maximize a particular objective. This technique, first described by James Kennedy and Russell C. Eberhart in 1995 [3], originates from two separateconcepts: the idea of swarm intelligence based off the observation of swarming habits by certainkinds ofanimals (such as birds and fish); and the field of evolutionary computation.

PSO belongs to the broad class of stochastic optimization algorithms. PSO is a population-based algorithm that exploits a population of individuals to probe promising regions of the search space. In this context, the population is called a swarm and the individuals are called particles. Each particle moves with an adaptable velocity within the search space, and retains in its memory the best position it ever encountered.

In the global variant of PSO the best position ever attained by all individuals of the swarm is communicated to all the particles. In the local variant, each particle is assigned to a neighborhood consisting of a prespecified number of particles.In this case, the best position ever attained by the particles that comprise the neighborhood is communicated among them.Finally, the PSO algorithm maintains the best fitness value achieved among all particles in the swarm, called the global best fitness, and the candidate solution that achieved this fitness, called the global best position or global best candidate solution. PSO also keeps the track of the all the best values that the particles have achieved so far.

\section{SYSTEM MODELING:}

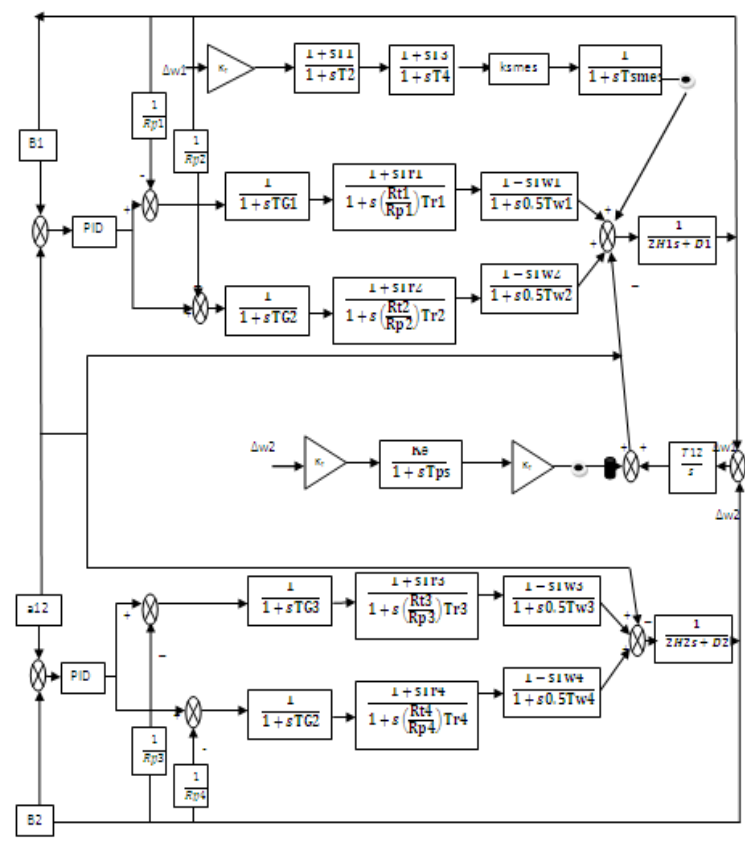

Fig3:linearised model of two area AGC system using SMES

(a) Linearised model of two area system using SMES:

In this we have considered a two area system with smes.The PID controller used in this system is being optimized using PSO,even the control variables of SMES system is optimized using PSO .For the dynamic performance of the test system $10 \%$ load change is considered.

(b)Mathematical model of SMES:

The structure of frequency stabilizer for SMES is modeled as the second order lead-lag compensator [ 4] and is shown in Fig. The objective of AGC is to reestablish primary frequencyregulation, restore the frequency to its nominal value as quickly as possible and minimize the tie-line power flows.In order to satisfy above requirements, the parameters of SMES are need to be optimized,which is done by using PSO. 


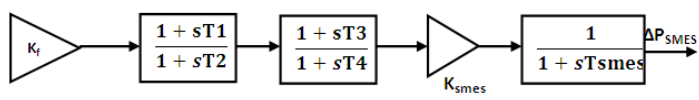

(c) Calculation of Area control error:

Fig.4 SMES Control scheme (ACE) to zero[2].

This error signal can be used to generate the Area Control Error (ACE) signal as:

$$
A C E_{i}=B_{i} \Delta f_{i}+\Delta P_{\text {tie-i-error }}
$$

Where, $\mathrm{Bi}$ is the frequency bias factor and $\Delta \mathrm{fi}$ is the frequency deviation in area-i.

\section{PSO WORKING:}

The PSO algorithm works by simultaneously maintaining several candidate solutions in the search space.During each iteration of the algorithm, each candidate solution is evaluated by the objective function being optimized, determining the fitness of that solution[9]. Each candidate solution can be thought of as a particle "flying" through the fitness landscape finding the maximum or minimum of the objective function. Initially, the PSO algorithm chooses candidate solutions randomly within the search space. Figure shows the initial state of a four-particle PSO algorithm seeking the global maximum in a one-dimensional search space[8].The PSO algorithm simply uses the objective function to evaluate itscandidate solutions, and operates upon the resultant fitness values.

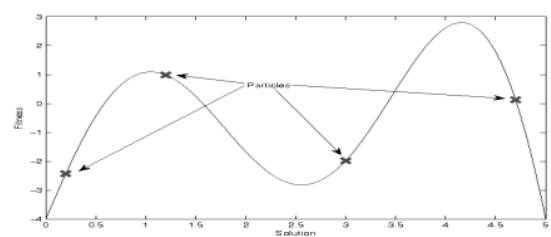

Fig5:Initial PSO State

Each particle maintains its position, composed of the candidate solution and its evaluated fitness, and its velocity. Additionally, it remembers the best fitness

value it has achieved,referred to as the individual bestposition or individual best candidate solution.The PSO algorithm consists of just three steps, which are repeated until some stopping condition is met

1. Evaluate the fitness of each particle

2. Update individual and global best fitnesses and positions

3. Update velocity and position of each particle

The first two steps are fairly trivial[7].

The velocity of each particle in the swarm is updated using the following equation[8]:

$\mathrm{v}_{\mathrm{i}}(\mathrm{t}+1)=\mathrm{w} \mathrm{v}_{\mathrm{i}}(\mathrm{t})+\mathrm{c}_{1} \mathrm{r}_{1}\left[\hat{\mathrm{X}}_{\mathrm{i}}(\mathrm{t})-\mathrm{x}_{\mathrm{i}}(\mathrm{t})\right]+\mathrm{c}_{2} \mathrm{r}_{2}\left[\mathrm{~g}(\mathrm{t})-\mathrm{x}_{\mathrm{i}}(\mathrm{t})\right]$

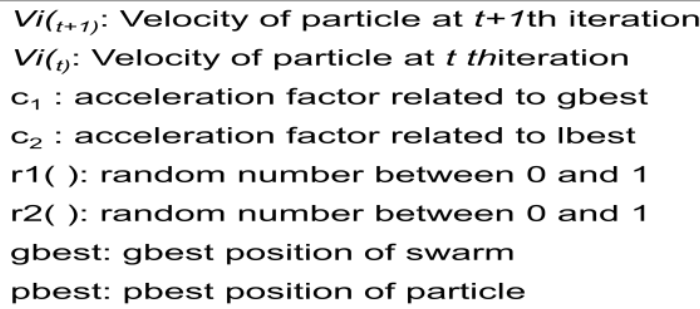




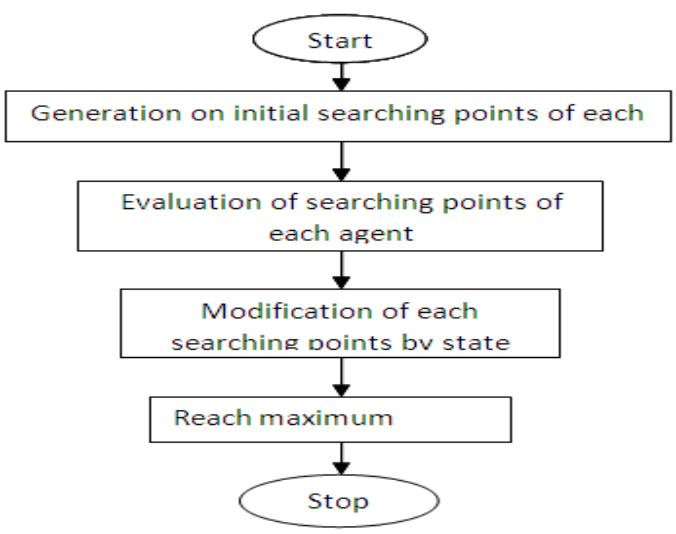

Fig6 - Flow Chart of particle swarm optimization

\section{Simulation ReSUlts:}

PSO based PID controller replaced the GA based PID controller,simulation result showed best solution.Further an SMES unit along with TCPS unit is introduced to the above system,its parameters have been optimized using PSO technique and we saw that an SMES\& TCPS based PSO PID controller gave much better result as compared to only SMES based PSO PID controler.

(a) Simulation Results of Two area system with \& without optimized TCPS :

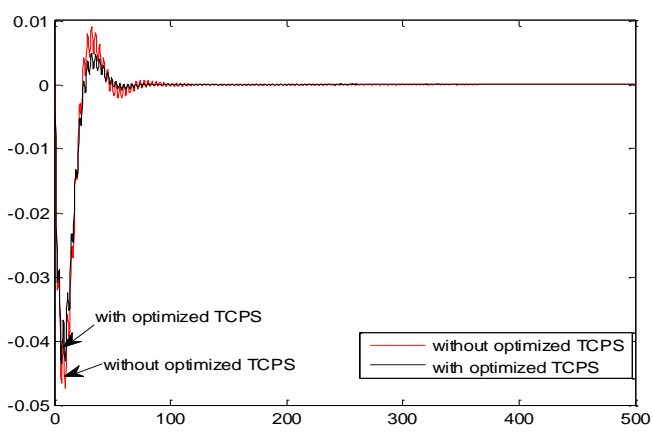

Fig7:Transient response of the test system with \& without TCPS

From the figure it can be seen that the system with optimized TCPS has less frequency deviation and much better dynamic stability as compared to the system without TCPS.

(b) Simulation Results of Two area system with optimized SMES without TCPS \& with optimized SMES \&TCPS

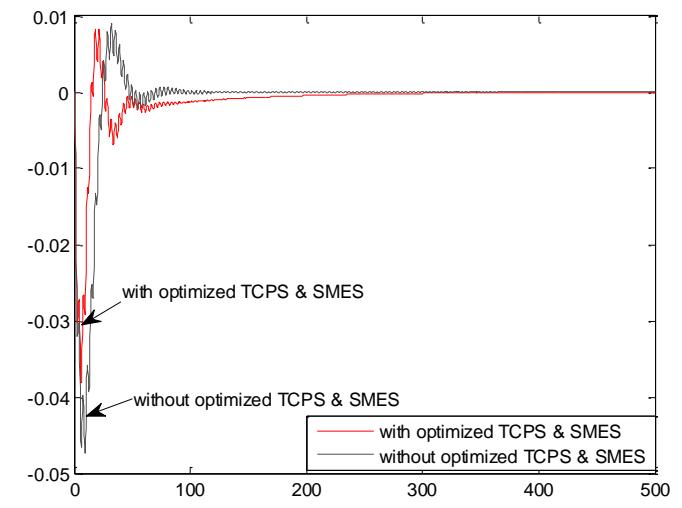

Fig8:Transient response of the test system with optimized SMES \& without TCPS \& with Optimized SMES \& $T C P S$

Transient response of the system with optimized TCPS \& SMES has better response and dynamic stability as compared to the system without TCPS and SMES.

(c) Comparision of Tieline power devition in two area system:

The Tie-line power deviation for test system without SMES and TCPS has been shown below. 


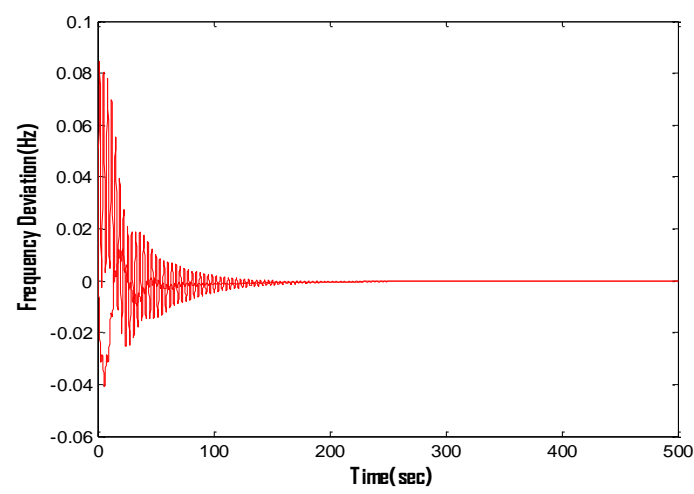

Fig9:Tielinedevition response of a PSO based Test system without optimized SMES\& TCPS

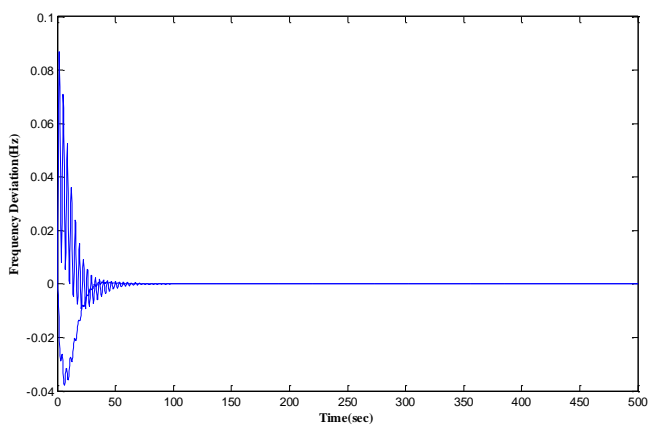

Fig10:Tie-line devition for test system with SMES and TCPS.

From Fig 9 \& 10 it can be seen that the settling time for the Tie line deviation for PSO based test system is much better as compared to GA based test system.

\section{CONCLUSION:}

Significant conclusion of this paper are as follows:

SMES coordination with TCPS can yield dynamic stability and effectively suppress the frequency and tie-line power oscillations after load disturbance and thus improve the transient response of the system.Application of TCPS coordinated with SMES may be successfully implemented for dynamic stabilization of dynamically unstable interconnected two-area multiple-units hydro-hydro system and for load frequency stabilization under load disturbance.PSO technique for the optimization of the parameters of PID controller,SMES \& TCPS yields better result as compared to the traditional optimization technique.

\section{REFERENCES}

[1] HasanBevrani\&TakashiHiyama,japan-IntelligentAutomatic Generation Control.Taylor\& francis gp publishing co. -2011

[2] Dr.SandeepBhongade,profH.O.Gupta senior member IEEE-Performance of SMES unit on Artificial Neural Network based multi area AGC scheme.journal of power electronics \& power system.Vol-1.March-2011.

[3] Mohd.Hassan Ali senior member IEEE-An overview of SMES application in energy \& power system, Vol. No.1-2010.

[4] Demiroren "Application to self tunning to Automatic Generation Control in power system including SMES unit" ,Newyork.2009

[5] S.P.GhoshalDept of Electrical Engg NIT Durgapur-Optimized AGC by SSSC \& TCPS in coordination with SMES for two area HydroHydro power system.International Conference on advances incomputing,Control\&telecommunicationtechnologie.2009

[6] James Blondin-Particle Swarm Optimization:A tutorial-sept 92009.

[7] FransVanderbergh-Analysis of particle swarm optimizers,University of Pretoria etd-2006

[8] Micheal N. Varhatis-On the computation of all global minimizers in Particle Swarm Optimization ,Vol 8,No.3 -2004

[9] James Kennedy and Russell Eberhart,-Particle swarm optimization,In Proceedings of the IEEE International Conference on Neural Networks, volume IV, pages 1942-1948, Piscataway, NJ, 1995.

[10] Yuhui Shi and Russell Eberhart, "A modified particle swarm optimizer", Proceedings of the IEEE International Conference on Evolutionary Computation, pages 69-73, 1998. 


\section{APPENDIX}

Table:The system data(capacity of each area is 1000MW)

\begin{tabular}{|l|l|}
\hline Pr1 $=$ Pr2 & $1000 \mathrm{MW}$ \\
\hline Kp1 $=$ Kp2 & $120 \mathrm{~Hz} / \mathrm{Pu} . \mathrm{MW}$ \\
\hline R1 $=$ R2 & $2.4 \mathrm{~Hz} / \mathrm{Pu} . \mathrm{MW}$ \\
\hline B1 $=$ B2 & 0.425 \\
\hline $\mathbf{D 1}=\mathbf{D 2}$ & 0.0083 \\
\hline Tp1 $=$ Tp2 & 205 \\
\hline T12 & 0.0707 \\
\hline Tg1 $=$ Tg2 & 0.2 \\
\hline $\mathbf{T}_{\mathrm{T} 1}=\mathbf{T}_{\mathrm{T} 2}$ & 0.3 \\
\hline
\end{tabular}

\begin{tabular}{|l|l|c|c|c|c|}
\hline \multicolumn{1}{|c|}{ OPTIMIZED SMES PARAMETERS } \\
\hline K$_{\text {SMES }}$ & $\mathbf{T}_{\text {SMES }}$ & T1 & T2 & T3 & T4 \\
\hline 0.2907 & 0.027 & 0.1119 & 0.8654 & 0.0132 & 0.1229 \\
\hline
\end{tabular}

\begin{tabular}{|c|c|}
\hline OPTIMIZED TCPS PARAMETERS \\
\hline $\mathrm{K} \phi=3.000$ & $\mathbf{T}_{\mathrm{PS}}=\mathbf{0 . 2 0 0}$ \\
\hline
\end{tabular}

